\title{
A systematic review and meta-analysis of genotype-based and individualized data analysis of SLCO1B1 gene and statin-induced myopathy
}

\author{
Saowalak Turongkaravee ${ }^{1} \cdot$ Jiraphun Jittikoon $\mathbb{1}^{2} \cdot$ Thitiya Lukkunaprasit $^{3} \cdot$ Sermsiri Sangroongruangsri ${ }^{4}$. \\ Usa Chaikledkaew ${ }^{4,5} \cdot$ Ammarin Thakkinstian $^{3,5}$
}

Received: 23 May 2020 / Revised: 5 December 2020 / Accepted: 15 January 2021 / Published online: 19 February 2021

(c) The Author(s) 2021. This article is published with open access

\begin{abstract}
This meta-analysis was conducted to determine the genotypic effects of rs4149056 and rs 2306283 polymorphism in SLCO1B1 gene on myopathy in patients with statin. Studies were searched using multiple databases and selected following inclusion criteria. Two reviewers independently performed data extraction and assessments for risk of bias. Fixed-orrandom-effect was applied to pool allele frequency/effects. Mixed-effect logit model was used to pool genotypic effects using individual patient data. Heterogeneity and publication bias were explored. Fourteen studies were pooled for rs4149056; the minor C allele frequency were $15 \%$ in Caucasians and 14\% in Asians. Six studies were pooled for rs 2306283; the minor G allele frequency was $34 \%$ in Caucasian and $75 \%$ in Asians. Genotypic effects of rs 4149056 polymorphism in Caucasians indicated that statin users who carried CC and TC genotypes had a significantly higher risk of myopathy than those who carried TT genotype, with a pooled odds ratio (OR) of 2.9 (95\% confidence interval, 1.59, 5.34) and $1.6(1.20,2.16)$, respectively. For subgroup analysis, CC and TC genotypes also suggested a higher risk of myopathy in simvastatin users $[\mathrm{OR}=2.8(1.17,6.77)$ and $\mathrm{OR}=1.8(1.15,2.77)$, respectively] and in atorvastatin users $[\mathrm{OR}=4.0(1.23$, $12.63)$ and $\mathrm{OR}=2.0(1.11,3.52)$, respectively] than those who carried TT genotype. There was no significant association between rs2306283 polymorphism and myopathy in Caucasians and Asians. There was no evidence of publication bias for both polymorphisms.
\end{abstract}

Supplementary information The online version contains supplementary material available at https://doi.org/10.1038/s41397021-00208-w.

Jiraphun Jittikoon

jiraphun.jit@mahidol.ac.th

1 Social, Economic and Administrative Pharmacy (SEAP) Graduate Program, Faculty of Pharmacy, Mahidol University,

Bangkok, Thailand

2 Department of Biochemistry, Faculty of Pharmacy, Mahidol University, Bangkok, Thailand

3 Department of Clinical Epidemiology and Biostatistics, Faculty of Medicine Ramathibodi Hospital, Mahidol University, Bangkok, Thailand

4 Social and Administrative Pharmacy Division, Department of Pharmacy, Faculty of Pharmacy, Mahidol University, Bangkok, Thailand

5 Mahidol University Health Technology Assessment (MUHTA) Graduate Program, Mahidol University, Bangkok, Thailand

\section{Introduction}

Hypercholesterolemia is a significant risk factor for cardiovascular diseases (CVDs) which is a vital cause of death in the world [1]. The mortality rate of CVDs is likely to increase in developing countries, especially in Asia [2] where the age-adjusted mortality rate was $82-215$ per 100,000 compared with $26-46$ per 100,000 in Western countries [3]. Statins, the lipid-lowering drugs class, are the first-line treatments for hypercholesterolemia that were recommended by the American College of Cardiology/ American Heart Association for the primary prevention of atherosclerotic cardiovascular disease in patients with lowdensity lipoprotein cholesterol (LDL-C) levels more than $190 \mathrm{mg} / \mathrm{dl}$ or diabetic patients [4]. Statins inhibit 3-hydroxy3-metylglutaryl-CoA reductase (HMG-CoA reductase) which could reduce LDL-C levels up to $55 \%$ and decrease CVDs by $20-30 \%$ [5].

However, myopathy commonly occurred in statin users, leading to poor adherence $[6,7]$. Its severity varied from 
myalgia toward life-threatening rhabdomyolysis [8]. The incidence of mild muscle pain, myopathy, and rhabdomyolysis was about 190, 11, and 3.4 per 100,000 patientyears [9], respectively. In Thailand, the Health Products Vigilance Center database of the Thai Food and Drug Administration from 2013 to 2017 reported that myopathy was listed in the top 20 adverse events caused by any statin [10]. The cause of myopathy is associated with receiving higher doses of statin or drug-interaction, which inhibit cytochrome P450 3A4 (i.e., gemfibrozil antifungals or macrolide antibiotics), or related to other risk factors (i.e., age, female, Asian descent, low body mass index, untreated hypothyroidism, or impaired renal/ hepatic function $[6,11,12])$. In addition, the genetic factors may play a role in statins-induced myopathy [13, 14]. Statin enters the systemic circulation through the influx transporter, the organic anion transporting polypeptide (OATP)1B1 encoded by the solute carrier organic anion transporter family member 1B1 gene (SLCO1B1) [15]. Two polymorphisms (rs2306283 and rs4149056) of SLCO1B1 gene have been reportedly associated with statin and risk of myopathy in statin users, as it could influence the plasma concentration of statins [15-18]. Accordingly, clinical practice guidelines from the Clinical Pharmacogenetics Implementation Consortium and the Royal Dutch Association for the Advancement of Pharmacy-Pharmacogenetics Working Group (DPWG) recommend that patients who carry the minor $\mathrm{C}$ allele (TC or $\mathrm{CC}$ ) of rs4149056 polymorphism may have an additional risk for simvastatin-induced myopathy. They should be prescribed a low dose of simvastatin (not exceeding $40 \mathrm{mg} /$ day), or an alternative statin (e.g., pravastatin or rosuvastatin), and a routine creatine kinase (CK) surveillance should be considered [15, 19]. Besides, DPWG also recommends that patients with TC or CC genotype of rs4149056 polymorphism may have a higher risk of atorvastatin-related myopathy, therefore alternate agents should be considered [19]. In addition, rs2306283 polymorphism of SLCO1B1 gene may potentially influence the pharmacokinetics and pharmacodynamics of statins [17, 20-22], because all statins are substrates of OATP1B1 transporter. Therefore, the effect of rs4149056 and rs2306283 polymorphism and myopathy in any statins should be investigated.

Recently, five meta-analyses (MA) [23-27], which included individual studies upto 2017, investigated the association between $S L C O 1 B 1$ gene and myopathy in statin users. They mainly focused on the SLCO1B1 (rs4149056) polymorphisms; only one MA also considered the rs2306283 polymorphisms in Caucasians [23]. However, these MAs did not include Asian studies and they pooled only minor allele effects or genetic model (i.e., recessive, co-dominant, dominant) rather than genotypic effects of each polymorphism in three genotypes (i.e., aa, Aa, and AA). Additionally, frequencies are different between the regions or population. For example, rs4149056 variant is the highest frequency in the native Americans and Europeans, whereas rs2306283 variant is the highest frequency in the Southeast Asian population [28, 29] which could affect the risk of myopathy in this population.

Therefore, this MA was conducted to assess the effect of these two polymorphisms of SLCO1B1 gene on myopathy in both Caucasian and Asian patients who received statin. In addition, a subgroup analysis was performed by statin types. Moreover, we also estimated both allele frequencies and mode of genetic effects of each polymorphism separately by ethnicity, as suggested by the guidelines of the Human Genome Epidemiology Network [30].

\section{Materials and methods}

This systematic review protocol was registered with PROSPERO, the International prospective register of systematic reviews (identification number CRD42018105282 and available from: http://www.crd.york.ac.uk/PROSPERO/display_ record.php?ID $=$ CRD42018105282). This review was conducted according to the guidelines of Preferred Reporting Items for Systematic Reviews and Meta-Analysis [31] and recently published guidance from the Human Genome Epidemiology Network for reporting gene-disease associations [30].

\section{Identification of studies}

The studies were located from the MEDLINE via PubMed and Scopus databases up to April 2019, based on search terms of gene (i.e., rs2306283 or rs4149056 within the SLCO1B1 genes) and outcome (i.e., myopathy) domains, see Supplementary Table S1. Searching was updated every 3 months, and the reference lists of relevant studies were also explored.

\section{Selection of studies}

Two reviewers (ST and SS) independently selected studies by screening titles and abstracts. If a decision for eligibility could be made, the full articles were reviewed. Any types of studies were included if they met all of the following criteria: (1) studied in general adults (aged 18 years or older) who received statin regardless indications, (2) studied two polymorphisms (i.e., rs2306283 or rs4149056) of the SLCO1B1 gene, and (3) had myopathy as the outcome. The studies were excluded if their data were insufficient for pooling and authors did not provide additional data after being contacted twice, as well as non-English studies. Any disagreements were resolved through consensus with the third author (TL). 


\section{Outcome of interest}

The primary outcome was myopathy defined by muscle weakness after statin use with/without confirming by $\mathrm{CK}$ higher rising three times, compared to the upper-normal limit [32].

\section{Data extraction}

Data were extracted independently by two reviewers (ST and SS) focusing on five parts, i.e., general information, study characteristics, patient characteristics, genes and outcome, and data for pooling, see Table 1 . If summary data were not reported, summary statistic data (i.e., odds ratio (OR)) were extracted. Authors were contacted if their reported data were insufficient for pooling. Disagreements were resolved by consensus with the third author (TL).

\section{Risk of bias assessment}

Two reviewers (ST and TL) independently assessed a risk of bias for genetic association studies [33] considering five domains: selection bias, information bias, confounding bias, multiple tests and selective outcome report, and Hardy-Weinberg equilibrium (HWE) assessment. Each item was classified as low/no ("yes"), possible/high ("no"), or "unclear" risk of bias. For any disagreement, this was resolved by the third author (SS).

\section{Statistical analysis}

Statistical analysis was performed following the method for meta-analysis of genetic association studies [34]. HWE was assessed in the control groups to detect equilibrium of two alleles (A and a, with frequencies $\mathrm{p}$ and $\mathrm{q}$, respectively) by using the Chi-square $\left(\chi^{2}\right)$ or exact test goodness-of-fit where appropriate. The studies were not included in pooling if they did not comply with HWE. Heterogeneity and its degree were assessed using Cochrane's $Q$ test and $I^{2}$ statistic. If the heterogeneity was present ( $p$ value $<0.1$ or $I^{2} \geq 25 \%$ ), the sources of heterogeneity were explored by meta-regression or subgroup analysis by potential factors (i.e., age groups, percent female, statin type, and duration use).

A minor allele frequency (MAF) in the control groups was pooled and stratified by ethnicity. Gene effects were estimated using two approaches (i.e., per allele and per genotype approaches). For the per allele approach, ORs of minor allele a vs major allele A along with $95 \%$ confidence interval (CI) were estimated. The ORs were pooled using a random-effect model by DerSimonian and Laird method [35] if heterogeneity was present ( $p$ value $<0.1$ or $I^{2} \geq 25 \%$ ); otherwise, a fixed-effect model with inverse variance method was applied. For the per genotype approach, the $\mathrm{OR}_{1}$ (aa vs $\mathrm{AA}$ ) and $\mathrm{OR}_{2}$ ( $\mathrm{Aa}$ vs $\mathrm{AA}$ ) were estimated using a mixed-effect logistic regression.

A sensitivity analysis was determined by including and excluding studies that did not comply with HWE in the main pooling to see robustness of the results based on a method for meta-analysis of genetic association studies [30, 34]. Lastly, publication bias was assessed using a funnel plot and the Egger's test [36]. A contour enhancedfunnel plot was constructed if any asymmetry was suggested by funnel plot or Egger's test [37]. All analyses were performed using STATA software version 15.0 (StataCorp, College Station, Texas, USA). A $p$ value $<0.05$ was considered as statistically significant.

\section{Results}

\section{Identifying of included studies}

A total of 2427 and 184 studies were, respectively, located from Scopus and MEDLINE via PubMed, but only 15 studies were eligible, see Fig. 1. Fourteen and six studies assessed effects of rs4149056 [17, 18, 26, 38-48] and rs2306283 polymorphisms [17, 38, 40, 41, 47, 49].

The characteristics of 15 included studies were demonstrated in Table 1. Of them, eight, five, and two were case-controls [26, 38, 40-43, 48, 49], cohorts [17, 39, 44, 46, 47], and randomized controlled trials $[18,45]$. Eleven, three, and one studies were conducted in Caucasians [17, 18, 26, 38-45], Asians [47-49], and mixed populations [46]. Eleven studies were investigated in patients receiving various statins $[17,26,38,39,41,42,44-46,48,49]$, the rests were simvastatin [18], atorvastatin [40], cerivastatin [43], and rosuvastatin only [47]. Mean age ranged from 53 to 74 years, and the proportion of males ranged from $33 \%$ to $83 \%$. The type of case was defined by muscle symptoms with/without CK confirmation.

\section{Risk of bias assessment}

The results of bias assessment are shown in Supplementary Table S2. All studies were clearly defined for diagnosis of myopathy and controls (non-myopathy). Therefore, they were low risk. However, nine studies $(60 \%)$ were unclear in quality control of genotyping. One study was unclear about the balance of ethnicity between case and control, which might be prone to population stratification.

Moreover, the risk of confounding bias might be presented in two studies that did not mention adjusting confounders. All studies demonstrated a significant association between SNPs and myopathy as a result of low risk of bias from selective outcome reporting. Ten of 15 studies (67\%) assessed HWE. 


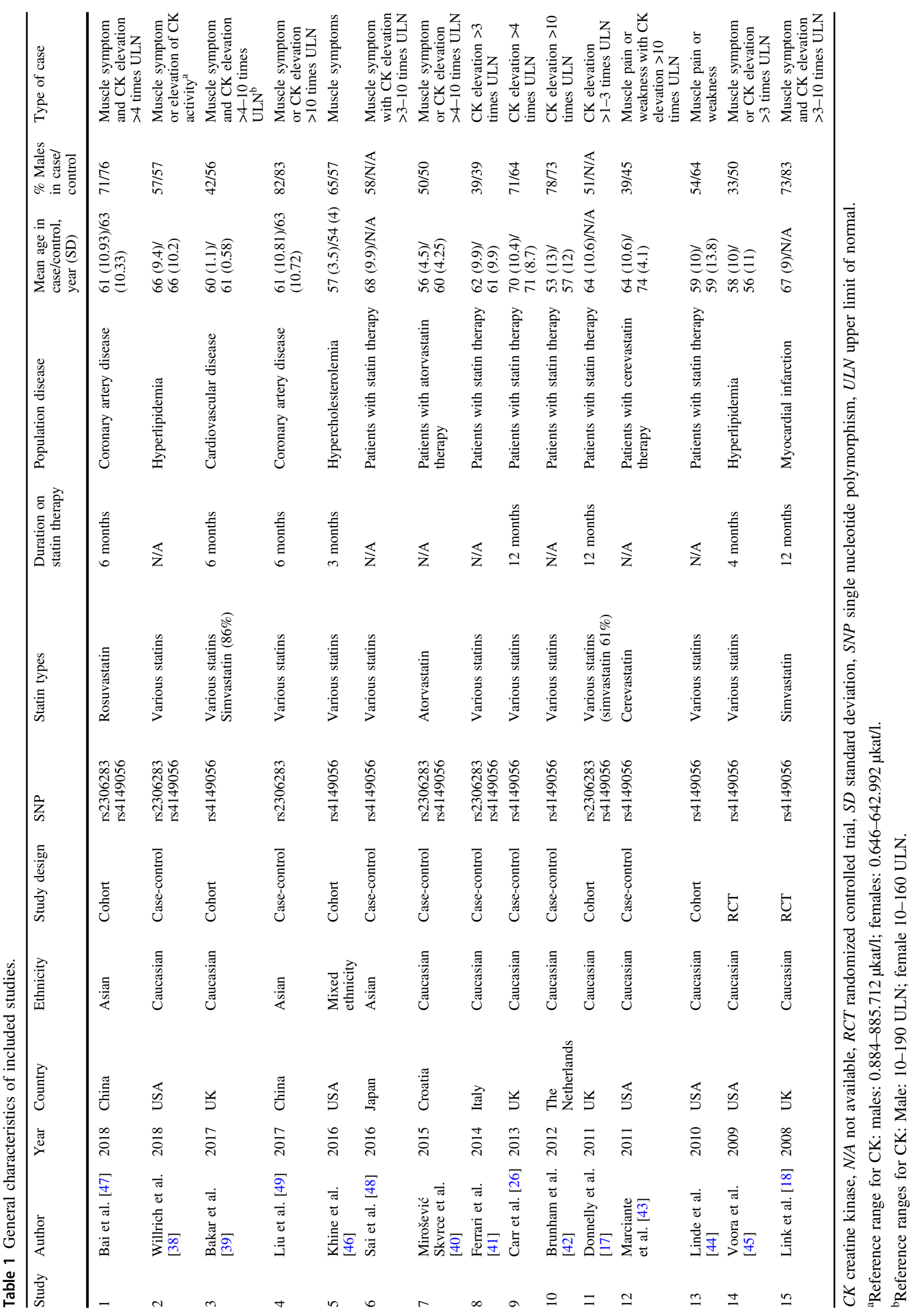




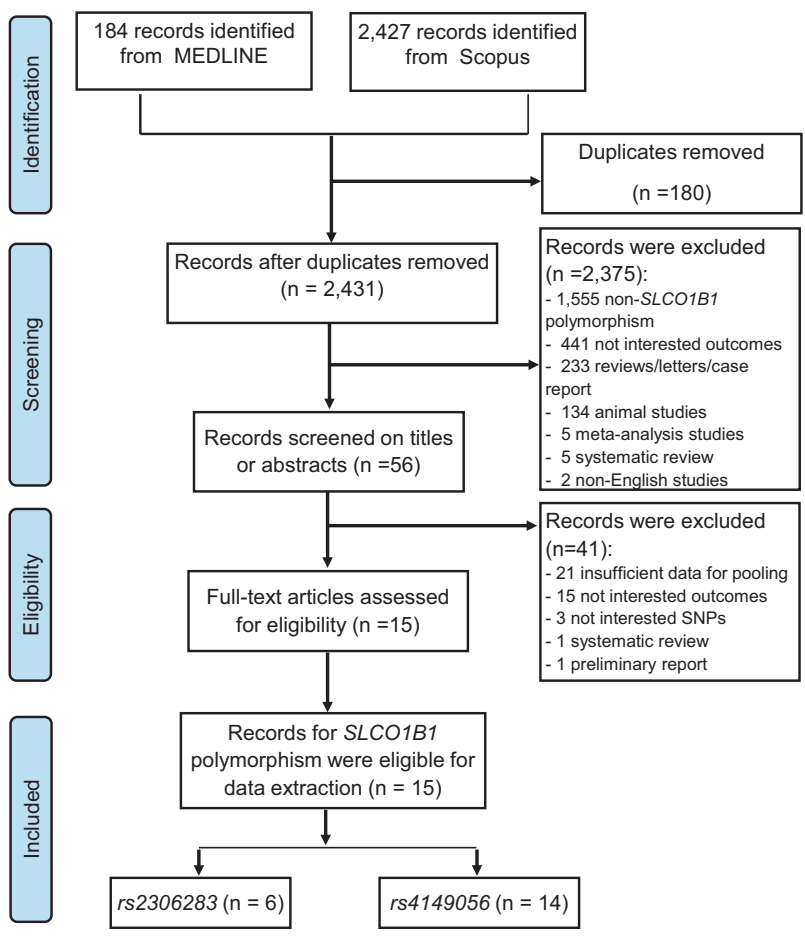

Fig. 1 PRISMA flow diagram of identifying and selecting studies for a systematic review and meta-analysis.

\section{Pooling results}

\section{rs4149056 polymorphism}

Fourteen studies investigated the association between rs4149056 and myopathy. Of these, 11 studies [17, 18, 26, 38-45] and two studies [47, 48] were conducted in Caucasians and Asians, respectively, while only one study investigated mixed ethnicity [46]. Ten studies investigated various statins with subgroup of statin types, i.e., atorvastatin, simvastatin, cerivastatin, and rosuvastatin. Allele frequencies across myopathy and control groups were described (Supplementary Table S3). All studies were compiled with the HWE rule. The MAF $\mathrm{C}$ among 11 Caucasian studies was higher in myopathy than that in control patients with the pooled frequencies $(95 \% \mathrm{CI})$ of $0.26(0.21,0.32)$ and 0.15 $(0.14,0.16)$, respectively. The allele effect was highly heterogeneous $\left(\chi^{2}=59.53, \mathrm{df}=10, p=<0.001, I^{2}=83.2 \%\right)$ with the pooled OR (C vs T) of 1.84 (1.35, 2.53). Therefore, a random-effect model was used to pool ORs across studies.

The pooled frequency of $\mathrm{C}$ allele in two Asian studies was $0.24(0.18,0.30)$ and $0.14(0.14,0.15)$ in case and control groups, respectively. However, the allele effect was homogeneous $\left(\chi^{2}=0.73\right.$, df $\left.=1, p=0.392, I^{2}=0 \%\right)$, with the pooled ORs $(95 \% \mathrm{CI})$ of $\mathrm{C}$ vs T of $1.87(1.34,2.60)$. A fixed-effect model was used to pool ORs across studies. The results suggested that statin users who carried minor $\mathrm{C}$ allele had $\sim 1.8$ times higher risk of myopathy than those who carried $\mathrm{T}$ allele in Caucasians and Asians. Sensitivity analysis was not performed, because all included studies were complied with HWE (which suggested that there was no indication of disequilibrium for all studies) and were included in the main pooling.

Genotype effects were estimated separately by ethnicity, see Table 2. For rs4149056 polymorphism, genotype effects were sufficient for pooling only in ten out of Caucasians studies [17, 18, 26, 38-42, 44, 45] ( $n=1433$ vs 2878 for myopathy vs control groups). Heterogeneity was present for both $\mathrm{OR}_{1} \quad\left(\mathrm{CC}\right.$ vs TT: $\chi^{2}=20.9, \quad \mathrm{df}=9, \quad p=0.013$, $\left.I^{2}=56.9 \%\right)$ and $\mathrm{OR}_{2} \quad\left(\mathrm{TC}\right.$ vs $\mathrm{TT}: \chi^{2}=32.7, \mathrm{df}=9$, $\left.p<0.001, I^{2}=72.5 \%\right)$. A mixed-effect logit model was used to pool $\mathrm{OR}_{1}$ and $\mathrm{OR}_{2}$ across studies, which yielded $\mathrm{OR}_{1}$ and $\mathrm{OR}_{2}$ of $2.9(1.59,5.34)$ and $1.6(1.20,2.16)$, respectively (Fig. 2A, B), indicating that statin users who carried CC and TC genotypes had 2.9 and 1.6 times significantly higher risk of myopathy than those statin users who carried TT genotype.

The potential sources of heterogeneity were explored by fitting age, female gender, duration of therapy, and the statins type in a meta-regression model (see Supplementary Table S6), indicating that statin type might be the source of heterogeneity. A subgroup analysis was performed accordingly. Genotype effects in atorvastatin users were homogeneous with the pooled $\mathrm{OR}_{1}$ and $\mathrm{OR}_{2}$ of 4.0 (1.23, 12.63) and 2.0 (1.11, 3.52), see Fig. 2C, D; whereas effects in simvastatin users were still heterogeneous with these corresponding ORs of $2.8(1.17,6.77)$ and $1.8(1.15,2.77)$, see Fig. 2E, F. Publication bias was assessed by funnel plot and Egger's test for pooled $\mathrm{OR}_{1}$ and $\mathrm{OR}_{2}$ in Caucasian with statin users, indicating no evidence of asymmetry of funnels for both ORs, see Supplementary Table S7 and Fig. S1.

\section{rs2306283 polymorphism}

Four and two studies assessed the association between rs2306283 and myopathy in Caucasians and Asians, and all studies complied with HWE. The pooled frequency of minor $\mathrm{G}$ allele in controls was a bit lower in Caucasians than that in Asians, i.e., $0.34(0.27,0.42)$ and 0.75 (0.74, $0.77)$, respectively. The ORs (G vs A) were 1.00 (0.71, $1.43)$ in Caucasians and $1.17(0.84,1.64)$ in Asians, see Supplementary Table S4. Sensitivity analysis was not performed, because all included studies were complied with HWE and were included in the main pooling.

Genotype effects were pooled among four Caucasians studies ( $n=998$ vs 1487 for myopathy vs control groups) and two Asian studies ( $n=199$ vs 956 for myopathy vs control groups), see Supplementary Table S5. The pooled $\mathrm{OR}_{1}$ (GG vs AA) and $\mathrm{OR}_{2}$ (AG vs AA) in statin users among Caucasians were $0.8(0.64,1.06)$ and $1.0(0.85,1.20)$ with moderate heterogeneity $I^{2}$ of $25.6 \%$ and $I^{2}=63.9 \%$, respectively (Fig. 3A, B). These corresponding ORs in statin users 


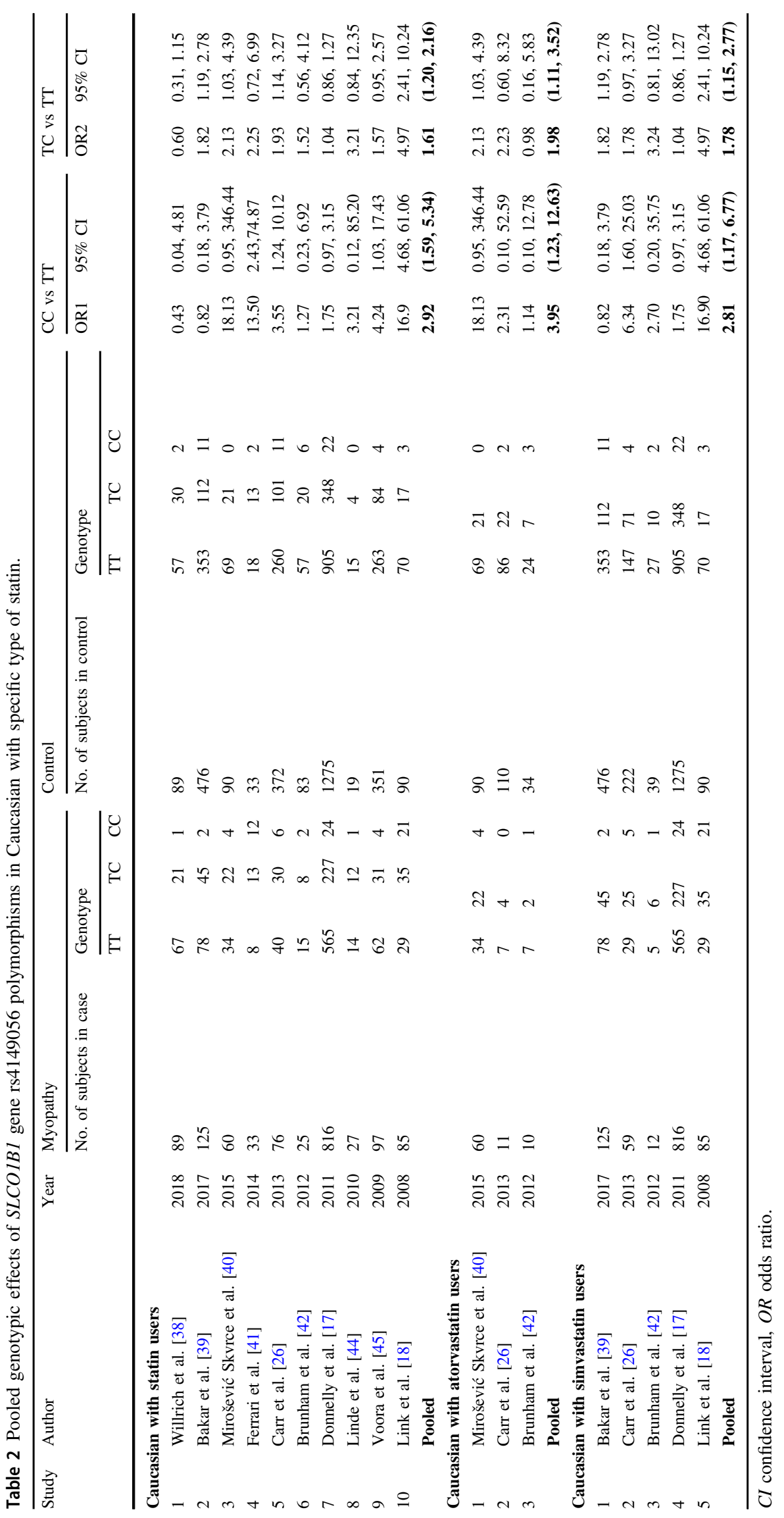




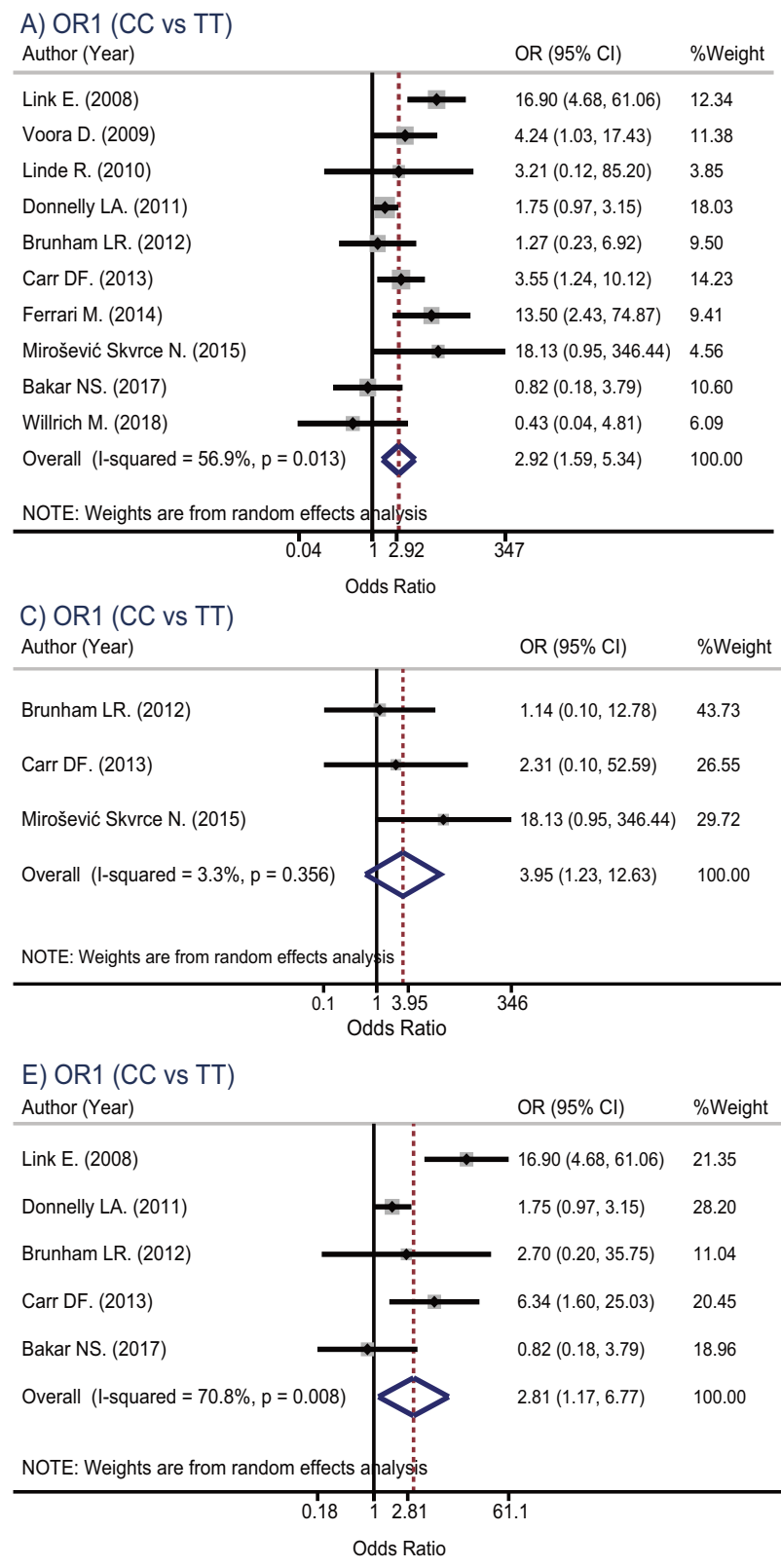

Fig. 2 Forest plots for pooling genotypic effects of $S L C O 1 B 1$ gene rs4149056 polymorphisms in Caucasians. $A \mathrm{OR}_{1}$ (CC vs TT) in statin users, $\mathbf{B} \mathrm{OR}_{2}$ (TC vs TT) in statin users, $\mathbf{C ~ O R}_{1}(\mathrm{CC}$ vs TT)

among Asians were $0.98(0.50,1.96)$ and $0.68(0.34,1.36)$ with the $I^{2}$ of $0.0 \%$ for both ORs (Fig. 3C, D).

Because the number of included studies was only four and two studies in Caucasians and Asians, respectively, the publication bias and meta-regression were not performed as the power of detecting was low.

\section{Discussion}

This meta-analysis of genetic association studies was conducted to assess the genetic effects of single nucleotide
B) OR2 (TC vs TT)

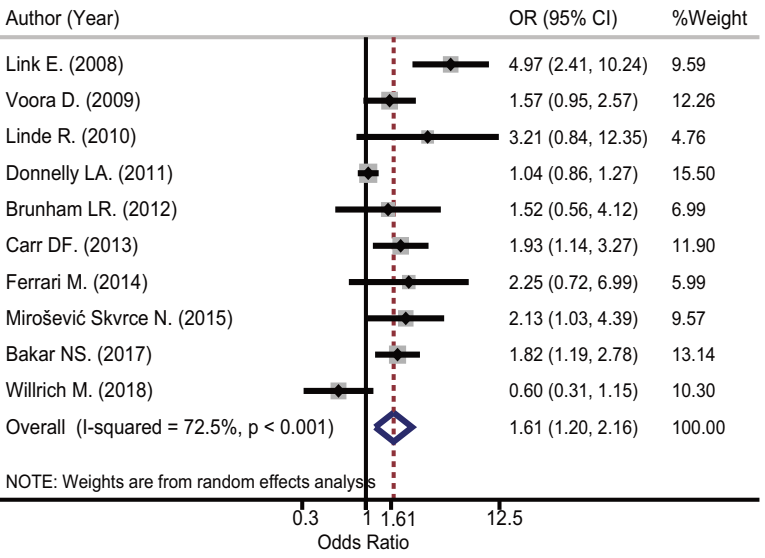

D) OR2 (TC vs TT)

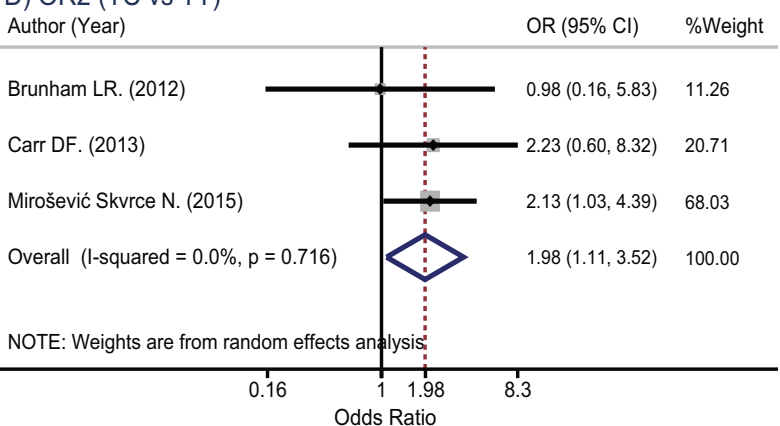

F) OR2 (TC vs TT)

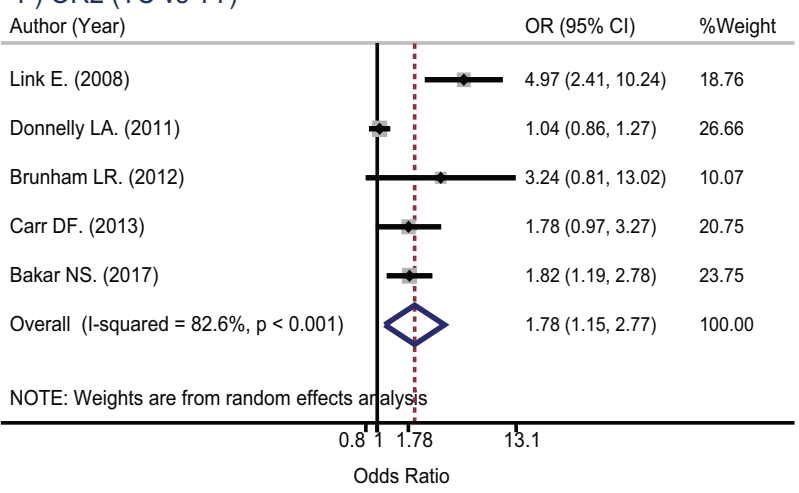

in atorvastatin users, $\mathbf{D} \mathrm{OR}_{2}$ (TC vs TT) in atorvastatin users, $\mathbf{E} \mathrm{OR}_{1}$ (CC vs TT) in simvastatin users, and $\mathbf{F} \mathrm{OR}_{2}$ (TC vs TT) in simvastatin users.

polymorphisms (rs4149056 and rs2306283) of the SLCO1BI gene on myopathy. Accordingly, the SLCO1BI gene is located on chromosome 12p12.1 (Online Mendelian Inheritance in Man \# 604843) [50]. Two polymorphisms (rs2306283 and rs4149056) of SLCO1B1 gene have been reported that they could influence statin and the risk of myopathy association [15-18]. Because the $S L C O 1 B 1$ gene is able to induce variations in plasma concentrations of statin through the influx transporter OATP1B1, it could influence the pharmacokinetic and pharmacodynamic profiles of statins [14, 51, 52].

Fourteen studies were included for rs4149056, and six studies for rs 2306283 in the main pooling. The frequency of 

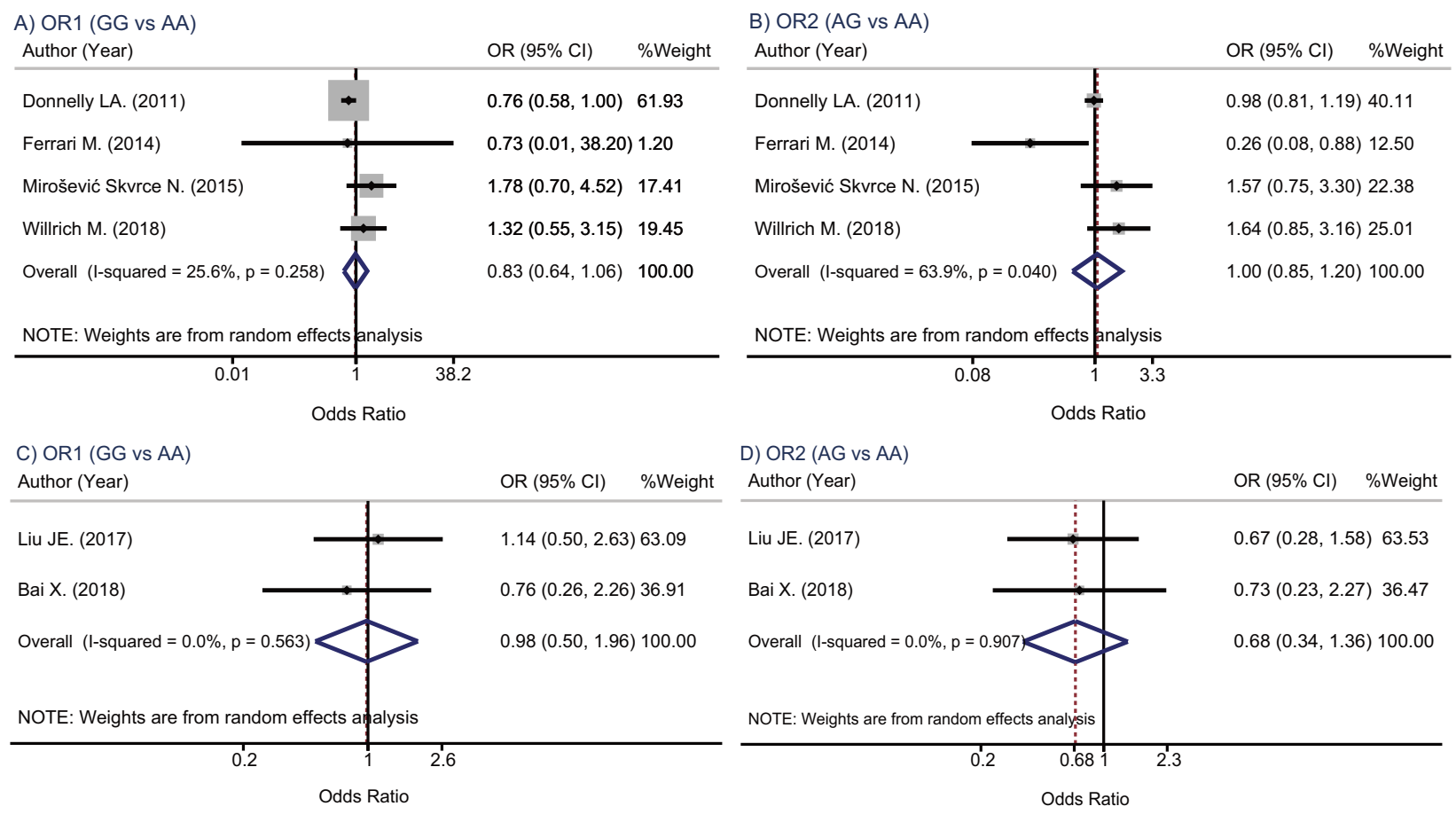

Fig. 3 Forest plots for pooling genotypic effects of $S L C O 1 B 1$ gene rs2306283 polymorphisms in statin users. A OR $(\mathrm{GG}$ vs $A A)$ in Caucasians, $\mathbf{B} \mathrm{OR}_{2}$ (AG vs AA) in Caucasians, $\mathbf{C}$ OR ${ }_{1}$ (GG vs AA) in Asians, $\mathbf{D} \mathrm{OR}_{2}$ (AG vs AA) in Asians.

the minor allele for each polymorphism was pooled and the magnitude of genotype effects was determined by ethnicity. The analysis was performed to include additional studies provided from previous evidence and complied with the guidelines of the Human Genome Epidemiology Network [30]. The finding suggested that the minor $\mathrm{C}$ allele frequencies for rs4149056 polymorphism in Caucasian were slightly higher than that in Asian ethnicities, yielding a frequency of $15 \%$ and $14 \%$, respectively. Indeed, the previous reviews reported that the $\mathrm{C}$ allele was associated with reduction of transport activity, which resulted in decreasing hepatic uptake and thus increasing plasma concentration of statins and a higher risk of myopathy. However, the effect is varied on the different types and dose of statins $[16,51,53,54]$. Likewise, our study contributes to robust associations in Caucasians, suggesting that individuals who were taking various types of statin therapies and carried the minor C allele, which were $\mathrm{CC}$ or $\mathrm{TC}$ genotypes of rs4149056 polymorphism, may have higher risks of myopathy by 2.9 and 1.6 times, respectively, than those who carried TT genotype. The results were consistent with previous MA [23-27], although there were different study designs with our study using mixed-effect logit model with individual patient data for pooling genotypic effects. Accordingly, we confirmed previous findings by including extensively more studies and demonstrated the genotype effects in three genotypes (i.e., aa, Aa, and AA), rather than assuming a specific genetic model (i.e., recessive, co-dominant, dominant) to avoid misleading estimates of the OR when an inappropriate model is assumed [36]. For subgroup analysis, which was stratified by statin types, the results suggested that individuals receiving specific types of statin (i.e., simvastatin and atorvastatin) had significant effect estimates similar to various statin among CC or TC genotypes carriers. Simvastatin users who were carrying CC or TC genotypes of rs 4149056 polymorphism had increased risks of myopathy by $\sim 2.8$ and 1.8 times, compared with those carrying the TT genotype. These findings were consistent with previous genome-wide association studies, which uncovered that carriers of CC and TC of rs4149056 had 16.9- and 4.5-times higher risk of myopathy than those of TT in users of simvastatin $80 \mathrm{mg}$ /daily [18]. However, our study did not perform a specific dosage of simvastatin because of insufficient data for pooling from original studies and authors did not provide additional data after being contacted twice. Furthermore, the risk of myopathy was higher in atorvastatin users by $\sim 4.0$ and 2.0 times among CC or TC genotypes carriers as compared with those who carried the TT genotype.

Our findings further supported the role of the SLCO1BI genotype in statin-associated myopathy and suggested that this association may be more robust for atorvastatin and simvastatin. There were some reasons suggesting that individuals who received atorvastatin may have higher risk of myopathy than those who took simvastatin. It may explain why patients who obtained higher equivalent doses 
of statins or more potent statins, in terms of lipid-lowering ability, may have an increasing tendency for myopathy risk. Consequently, included studies on atorvastatin used dosage between 20 and $80 \mathrm{mg}$ daily, which was classified as a moderate to high-intensity therapy. On the other hand, included studies on simvastatin mostly used $10-40 \mathrm{mg}$ daily, which was a low to moderate-intensity therapy. Nevertheless, we did not report a specific dosage for any statin because of insufficient data for pooling from the original studies.

In addition, we performed TC $+\mathrm{CC}$ vs TT of rs4149056 polymorphism among ten studies in Caucasians. The results showed that individuals who carried TC $+\mathrm{CC}$ genotype also had a higher risk of myopathy when compared with TT genotype in statin users, with a pooled OR of 1.9 (95\% CI: $1.28,2.83 ; p<0.001)$, and simvastatin users 2.2 (1.2, 4.2; $p<0.001)$ and atorvastatin users $2.1(1.2,3.8 ; p=0.585)$. Our finding is similar to Xiang et al. [27]. The results suggested that patients who carrying the minor $\mathrm{C}$ allele (i.e., $\mathrm{TC}, \mathrm{CC}$ or $\mathrm{TC}+\mathrm{CC}$ ) may have an additional risk of myopathy when compared with TT genotype. However, we included additional studies provided from previous evidence and there were different study designs with our study using mixed-effect logit model with individual patient data for pooling.

For the rs2306283, our study showed that the minor $G$ allele frequencies were common in Asians, but rare in Caucasians, yielding a frequency of $75 \%$ in Asians and $34 \%$ in Caucasians. Nevertheless, this study suggested that there was no significant association between rs2306283 polymorphism and myopathy in Asians and Caucasians with statin users. It would be probably caused by the small numbers of included studies. Furthermore, the role of rs2306283 polymorphism in the transporter function was still controversial. For instance, the $\mathrm{G}$ allele might increase plasma concentration and cause myotoxicity [55]. On the other hand, it might increase transporter activity resulting in the lower oral bioavailability or decreased plasma concentration of statin and pravastatin $[14,56,57]$, while the latter could be an attenuation of lower risk of statin-induced myopathy in patients who carried GG or AG genotypes [17]. Therefore, we would need more association studies in both Caucasian and Asian populations to confirm our findings.

\section{Strengths and limitations of the study}

Our study had several strengths. Data analysis was performed following a standard method for meta-analysis of genetic association studies [30, 34]. We also pooled allele frequency and genetic effects separately, which was a requirement of the Human Genome Epidemiology Network [30]. Moreover, genotype effects were pooled using a genetic model-free approach with more studies in both rs4149056 and rs2306283 polymorphisms, compared with previous evidence, especially in the Asian population. Furthermore, the gene effects were estimated from individual patient data of genotypes and outcome of individual studies. Our study applied a mixed-effect logistic regression and separately by ethnicity to fit genotypes. The finding could be generalized as compared with previous studies because it is the first meta-analysis that included Asian studies given that the SLCO1B1 gene is generally distributed and different between regions [28].

Moreover, the included studies had a definite diagnosis of myopathy case and control groups and all studies complied with HWE in the main pooling as a result of limiting potential bias in outcome measures. Indeed, HWE is a mathematical equation that can be used to calculate the genetic variation of a population at equilibrium as well as provides a quality of genetic association studies in terms of design and conduct. The deviation from HWE in the control group may be due to selection bias, population stratification and genotyping errors, which may bias the estimates of genetic effects [58-60].

Besides, our study reported no evidence of publication bias due to small study effects. Moreover, sources of heterogeneity were explored. However, other characteristics may cause heterogeneity, because either studies did not report from original studies (i.e., co-medication, a dosage of administration, hypothyroidism, chronic kidney disease, excess alcohol intake) or the data were not sufficient for pooling.

Given that all types of statins were substrates of OATP1B1 transporter, we would need more genetic association studies of the SLCO1B1 gene and myopathy among other types of statin to confirm that the effects could be a class effect or specific types of statin only. Future research including analysis cost-effectiveness study on the SLCO1B1 gene is also required. It may be useful for assessing the value of money of pharmacogenetic testing before prescribing statin, preventing the adverse events, and improving patient's adherence considering that pharmacogenetic testing plays an extensive role on precision and personalized medicine.

\section{Policy recommendation}

This study provided an understanding of the roles of genetic and drug response. Our findings may be considered an extension of traditional approaches to treat a disease, which is useful for physicians, as they could select a drug treatment or intervention based on a patient's molecular profile. This could lead to a reduction of harmful adverse effects, ensuring an effective outcome as well as containing costs from a "trial-and-error" approach to disease treatment. 
Moreover, policy-makers could make evidence-based decisions for the selection of drugs or alternative drugs on a list of Thai National List of Essential Medicines for patients through the genetic information added to the clinical and economic evidence.

\section{Conclusions}

In summary, this meta-analysis provides a robust association in Caucasians indicating that individuals who carried the minor C allele, with CC or TC genotypes of rs 4149056 polymorphism may particularly have higher risks of myopathy among various statin users and especially in those who received simvastatin and atorvastatin, compared with those who carried the TT genotype. No significant association between rs 2306283 polymorphism and myopathy was caused by various statin users was found in Asian and Caucasian populations.

Acknowledgements This work is a part of a study in Doctor of Philosophy program in Social, Economic and Administrative Pharmacy (SEAP), Faculty of Pharmacy, Mahidol University, Bangkok. Besides, this study was supported by grants from the International Research Network - the Thailand Research Fund (IRN60W003) and Health Systems Research Institute (HSRI61-080), Thailand. However, the funders had no role in the design, conduct of the study; collection, management, analysis, and interpretation of data; and preparation of the manuscript.

\section{Compliance with ethical standards}

Conflict of interest The authors declare that they have no conflict of interest.

Publisher's note Springer Nature remains neutral with regard to jurisdictional claims in published maps and institutional affiliations.

Open Access This article is licensed under a Creative Commons Attribution 4.0 International License, which permits use, sharing, adaptation, distribution and reproduction in any medium or format, as long as you give appropriate credit to the original author(s) and the source, provide a link to the Creative Commons license, and indicate if changes were made. The images or other third party material in this article are included in the article's Creative Commons license, unless indicated otherwise in a credit line to the material. If material is not included in the article's Creative Commons license and your intended use is not permitted by statutory regulation or exceeds the permitted use, you will need to obtain permission directly from the copyright holder. To view a copy of this license, visit http://creativecommons. org/licenses/by/4.0/.

\section{References}

1. World Health Organization. Global Health Estimates 2016: disease burden by causbalare, age, sex, by country and by region, 2000-2016. Geneva: World Health Organization; 2018.
2. Hong Y. Burden of cardiovascular disease in asia: big challenges and ample opportunities for action and making a difference. Clin Chem. 2009;55:1450.

3. WHO Statistical Information System. Causes of death: mortality and health status. WHO data and statistics. http://www.who.int/ research/en/. Accessed 21 Nov 2019.

4. Correction to: 2019 ACC/AHA guideline on the primary prevention of cardiovascular disease: a report of the American College of Cardiology/American Heart Association Task Force on clinical practice guidelines. Circulation. 2019;140:e649-50.

5. Baigent C, Blackwell L, Emberson J, Holland LE, Reith C, Bhala $\mathrm{N}$, et al. Efficacy and safety of more intensive lowering of LDL cholesterol: a meta-analysis of data from 170,000 participants in 26 randomised trials. Lancet. 2010;376:1670-81.

6. Rallidis LS, Fountoulaki K, Anastasiou-Nana M. Managing the underestimated risk of statin-associated myopathy. Int J Cardiol. 2012;159:169-76.

7. Mampuya WM, Frid D, Rocco M, Huang J, Brennan DM, Hazen SL, et al. Treatment strategies in patients with statin intolerance: the Cleveland Clinic experience. Am Heart J. 2013;166:597-603.

8. Pasternak RC, Smith SC Jr., Bairey-Merz CN, Grundy SM, Cleeman JI, Lenfant C. ACC/AHA/NHLBI clinical advisory on the use and safety of statins. Stroke. 2002;33:2337-41.

9. Law M, Rudnicka AR. Statin safety: a systematic review. Am J Cardiol. 2006;97:52c-60c.

10. Health Product Vigilance Center. Spontaneous reports of ADRs. Nonthaburi: Thai Food and Drug Administration, Ministry of Public Health; 2017.

11. Sathasivam S. Statin induced myotoxicity. Eur J Intern Med. 2012;23:317-24.

12. Sathasivam S, Lecky B. Statin induced myopathy. BMJ. 2008;337:a2286.

13. Kitzmiller JP, Binkley PF, Pandey SR, Suhy AM, Baldassarre D, Hartmann K. Statin pharmacogenomics: pursuing biomarkers for predicting clinical outcomes. Disco Med. 2013;16:45-51.

14. Arrigoni E, Del ReM, Fidilio L, Fogli S, Danesi R, Di Paolo A. Pharmacogenetic foundations of therapeutic efficacy and adverse events of statins. Int J Mol Sci. 2017;18:104.

15. Wilke RA, Ramsey LB, Johnson SG, Maxwell WD, McLeod HL, Voora D, et al. The clinical pharmacogenomics implementation consortium: CPIC guideline for SLCO1B1 and simvastatininduced myopathy. Clin Pharm Ther. 2012;92:112-7.

16. Tirona RG, Leake BF, Merino G, Kim RB. Polymorphisms in OATP-C: identification of multiple allelic variants associated with altered transport activity among European- and AfricanAmericans. J Biol Chem. 2001;276:35669-75.

17. Donnelly LA, Doney AS, Tavendale R, Lang CC, Pearson ER, Colhoun HM, et al. Common nonsynonymous substitutions in SLCO1B1 predispose to statin intolerance in routinely treated individuals with type 2 diabetes: a go-DARTS study. Clin Pharm Ther. 2011;89:210-6.

18. Link E, Parish S, Armitage J, Bowman L, Heath S, Matsuda F, et al. SLCO1B1 variants and statin-induced myopathy-a genomewide study. N Engl J Med. 2008;359:789-99.

19. The Royal Dutch Pharmacists Association Pharmacogenetics Working Group. Annotation of DPWG Guideline for atorvastatin and SLCO1B1. 2019. https://www.knmp.nl/downloads/pharma cogenetic-recommendations-august-2019.pdf. Accessed 21 Nov 2019.

20. Santos PC, Gagliardi AC, Miname MH, Chacra AP, Santos RD, Krieger JE, et al. SLCO1B1 haplotypes are not associated with atorvastatin-induced myalgia in Brazilian patients with familial hypercholesterolemia. Eur J Clin Pharm. 2012;68:273-9.

21. Melo MS, Balanco L, Branco CC, Mota-Vieira L. Genetic variation in key genes associated with statin therapy in the Azores 
Islands (Portugal) healthy population. Ann Hum Biol. 2015;42: 283-9.

22. Giannakopoulou E, Ragia G, Kolovou V, Tavridou A, Tselepis $\mathrm{AD}$, Elisaf M, et al. No impact of SLCO1B1 521T>C, 388A $>\mathrm{G}$ and $411 \mathrm{G}>\mathrm{A}$ polymorphisms on response to statin therapy in the Greek population. Mol Biol Rep. 2014;41:4631-8.

23. Jiang J, Tang Q, Feng J, Dai R, Wang Y, Yang Y, et al. Association between SLCO1B1-521T>C and $-388 \mathrm{~A}>\mathrm{G}$ polymorphisms and risk of statin-induced adverse drug reactions: a meta-analysis. SpringerPlus. 2016;5:1368.

24. Hou Q, Li S, Li L, Li Y, Sun X, Tian H. Association between SLCO1B1 Gene T521C polymorphism and statin-related myopathy risk: a meta-analysis of case-control studies. Medicine (Baltimore). 2015;94:e1268.

25. De Keyser CE, Peters BJM, Becker ML, Visser LE, Uitterlinden AG, Klungel $\mathrm{OH}$, et al. The SLCO1B1 c.521T>C polymorphism is associated with dose decrease or switching during statin therapy in the Rotterdam Study. Pharmacogenet Genomics. 2014;24: $43-51$.

26. Carr DF, O'Meara H, Jorgensen AL, Campbell J, Hobbs M, McCann G, et al. SLCO1B1 genetic variant associated with statininduced myopathy: a proof-of-concept study using the clinical practice research datalink. Clin Pharm Ther. 2013;94:695-701.

27. Xiang Q, Chen SQ, Ma LY, Hu K, Zhang Z, Mu GY, et al. Association between SLCO1B1 T521C polymorphism and risk of statin-induced myopathy: a meta-analysis. Pharmacogenomics J. 2018;18:721-9.

28. Pasanen MK, Neuvonen PJ, Niemi M. Global analysis of genetic variation in. SLCO1B1 Pharmacogenomics. 2008;9:19-33.

29. Jittikoon J, Mahasirimongkol S, Charoenyingwattana A, Chaikledkaew U, Tragulpiankit P, Mangmool S, et al. Comparison of genetic variation in drug ADME-related genes in Thais with Caucasian, African and Asian HapMap populations. J Hum Genet. 2016;61:119-27.

30. Little JHJ, eds. The HuGENet ${ }^{\mathrm{TM}}$ HuGE review handbook, Version 1.0. Ottawa, Ontario, Canada: University of Ottawa; 2006.

31. Moher D, Liberati A, Tetzlaff J, Altman DG. Preferred reporting items for systematic reviews and meta-analyses: the PRISMA statement. J Clin Epidemiol. 2009;62:1006-12.

32. Thompson PD, Panza G, Zaleski A, Taylor B. Statin-associated side effects. J Am Coll Cardiol. 2016;67:2395.

33. Thakkinstian A, McKay GJ, McEvoy M, Chakravarthy U, Chakrabarti S, Silvestri G, et al. Systematic review and metaanalysis of the association between complement component 3 and age-related macular degeneration: a HuGE review and metaanalysis. Am J Epidemiol. 2011;173:1365-79.

34. Thakkinstian A, McElduff P, D'Este C, Duffy D, Attia J. A method for meta-analysis of molecular association studies. Stat Med. 2005;24:1291-306.

35. DerSimonian R, Laird N. Meta-analysis in clinical trials. Control Clin Trials. 1986;7:177-88.

36. Minelli C, Thompson JR, Abrams KR, Thakkinstian A, Attia J. The choice of a genetic model in the meta-analysis of molecular association studies. Int J Epidemiol. 2005;34:1319-28.

37. Egger M, Davey Smith G, Schneider M, Minder C. Bias in metaanalysis detected by a simple, graphical test. BMJ. 1997;315:629-34.

38. Willrich MAV, Kaleta EJ, Bryant SC, Spears GM, Train LJ, Peterson SE, et al. Genetic variation in statin intolerance and a possible protective role for UGT1A1. Pharmacogenomics. 2018; 19:83-94.

39. Bakar NS, Neely D, Avery P, Brown C, Daly AK, Kamali F. Genetic and clinical factors are associated with statin-related myotoxicity of moderate severity: a case-control study. Clin Pharm Ther. 2017;104:178-87.
40. Mirošević Skvrce N, Macolić Šarinić V, Šimić I, Ganoci L, Muačević Katanec D, Božina N. ABCG2 gene polymorphisms as risk factors for atorvastatin adverse reactions: a case-control study. Pharmacogenomics. 2015;16:803-15.

41. Ferrari M, Guasti L, Maresca A, Mirabile M, Contini S, Grandi $\mathrm{AM}$, et al. Association between statin-induced creatine kinase elevation and genetic polymorphisms in SLCO1B1, ABCB1 and ABCG2. Eur J Clin Pharm. 2014;70:539-47.

42. Brunham LR, Lansberg PJ, Zhang L, Miao F, Carter C, Hovingh GK, et al. Differential effect of the rs4149056 variant in SLCO1B1 on myopathy associated with simvastatin and atorvastatin. Pharmacogenomics J. 2012;12:233-7.

43. Marciante KD, Durda JP, Heckbert SR, Lumley T, Rice K, McKnight B, et al. Cerivastatin, genetic variants, and the risk of rhabdomyolysis. Pharmacogenet Genomics. 2011;21:280-8.

44. Linde R, Peng L, Desai M, Feldman D. The role of vitamin D and $S L C O 1 B 1 * 5$ gene polymorphism in statin-associated myalgias. Dermatoendocrinol. 2010;2:77-84.

45. Voora D, Shah SH, Spasojevic I, Ali S, Reed CR, Salisbury BA, et al. The $S L C O 1 B 1 * 5$ genetic variant is associated with statininduced side effects. J Am Coll Cardiol. 2009;54:1609-16.

46. Khine H, Yuet WC, Adams-Huet B, Ahmad Z. Statin-associated muscle symptoms and SLCO1B1 rs4149056 genotype in patients with familial hypercholesterolemia. Am Heart J. 2016;179:1-9.

47. Bai X, Zhang B, Wang P, Wang GL, Li JL, Wen DS, et al. Effects of SLCO1B1 and GATM gene variants on rosuvastatin-induced myopathy are unrelated to high plasma exposure of rosuvastatin and its metabolites. Acta Pharm. 2018;40:492-9.

48. Sai K, Kajinami K, Akao H, Iwadare M, Sato-Ishida R, Kawai Y, et al. A possible role for HLA-DRB $1 * 04: 06$ in statin-related myopathy in Japanese patients. Drug Metab Pharmacokinet. 2016;31:467-70.

49. Liu JE, Liu XY, Chen S, Zhang Y, Cai LY, Yang M, et al. SLCO1B1 521T $>$ C polymorphism associated with rosuvastatininduced myotoxicity in Chinese coronary artery disease patients: a nested case-control study. Eur J Clin Pharm. 2017;73:1409-16.

50. McKusick’s Online Mendelian Inheritance in Man OMIM ${ }^{\circledR} .2019$. http://www.ncbi.nlm.nih.gov/omim. Accessed 30 June 2019.

51. Niemi M, Pasanen MK, Neuvonen PJ. Organic anion transporting polypeptide 1B1: a genetically polymorphic transporter of major importance for hepatic drug uptake. Pharm Rev. 2011;63:157-81.

52. Niemi M, Schaeffeler E, Lang T, Fromm MF, Neuvonen M, Kyrklund $\mathrm{C}$, et al. High plasma pravastatin concentrations are associated with single nucleotide polymorphisms and haplotypes of organic anion transporting polypeptide-C (OATP-C, SLCO1B1). Pharmacogenetics. 2004;14:429-40.

53. Kameyama Y, Yamashita K, Kobayashi K, Hosokawa M, Chiba K. Functional characterization of $S L C O 1 B 1(O A T P-C)$ variants, $S L C O 1 B 1 * 5, \quad S L C O 1 B 1 * 15$ and $S L C O 1 B 1 * 15+\mathrm{C} 1007 \mathrm{G}$, by using transient expression systems of HeLa and HEK293 cells. Pharmacogenet Genomics. 2005;15:513-22.

54. Nozawa T, Nakajima M, Tamai I, Noda K, Nezu J, Sai Y, et al. Genetic polymorphisms of human organic anion transporters OATP-C (SLC21A6) and OATP-B (SLC21A9): allele frequencies in the Japanese population and functional analysis. J Pharm Exp Ther. 2002;302:804-13.

55. DeGorter MK, Tirona RG, Schwarz UI, Choi YH, Dresser GK, Suskin N, et al. Clinical and pharmacogenetic predictors of circulating atorvastatin and rosuvastatin concentrations in routine clinical care. Circ Cardiovasc Genet. 2013;6:400-8.

56. Mwinyi J, Johne A, Bauer S, Roots I, Gerloff T. Evidence for inverse effects of OATP-C (SLC21A6) 5 and 1b haplotypes on pravastatin kinetics. Clin Pharm Ther. 2004;75:415-21.

57. Maeda K, Ieiri I, Yasuda K, Fujino A, Fujiwara H, Otsubo K, et al. Effects of organic anion transporting polypeptide 1B1 
haplotype on pharmacokinetics of pravastatin, valsartan, and temocapril. Clin Pharm Ther. 2006;79:427-39.

58. Thakkinstian A, McEvoy M, Chakravarthy U, Chakrabarti S, McKay GJ, Ryu E, et al. The association between complement component $2 /$ complement factor B polymorphisms and agerelated macular degeneration: a HuGE review and meta-analysis. Am J Epidemiol. 2012;176:361-72.
59. Zintzaras E, Lau J. Synthesis of genetic association studies for pertinent gene-disease associations requires appropriate methodological and statistical approaches. J Clin Epidemiol. 2008;61: 634-45.

60. Namipashaki A, Razaghi-Moghadam Z, Ansari-Pour N. The essentiality of reporting Hardy-Weinberg equilibrium calculations in population-based genetic association studies. Cell J. 2015;17:187-92. 